\title{
Therapeutic potential of targeting S100A11 in malignant pleural mesothelioma
}

\author{
Hiroki Sato ${ }^{1}$, Masakiyo Sakaguchi ${ }^{2}$, Hiromasa Yamamoto (1)', Shuta Tomida ${ }^{3}$, Keisuke Aoe ${ }^{4,5}$, Kazuhiko Shien (1)', \\ Takahiro Yoshioka ${ }^{6}$, Kei Namba' ${ }^{1}$ Hidejiro Torigoe ${ }^{1}$, Junichi Soh' ${ }^{1}$, Kazunori Tsukuda ${ }^{1}$, Hiroyuki Tao ${ }^{5,7}$, Kazunori Okabe ${ }^{5,7}$, \\ Shinichiro Miyoshi ${ }^{1}$, Harvey I. Pass ${ }^{8}$ and Shinichi Toyooka ${ }^{1,6}$
}

\begin{abstract}
Malignant pleural mesothelioma (MPM) is an aggressive tumor with an unfavorable prognosis. The standard therapeutic approaches are limited to surgery, chemotherapy, and radiotherapy. Because the consequent clinical outcome is often unsatisfactory, a different approach in MPM treatment is required. S100A11, a $\mathrm{Ca}^{2+}$-binding small protein with two EF-hands, is frequently upregulated in various human cancers. Interestingly, it has been found that intracellular and extracellular S100A11 have different functions in cell viability. In this study, we focused on the impact of extracellular S100A11 in MPM and explored the therapeutic potential of an S100A11-targeting strategy. We examined the secretion level of S100A11 in various kinds of cell lines by enzyme-linked immunosorbent assay. Among them, six out of seven MPM cell lines actively secreted S100A11, whereas normal mesothelial cell lines did not secrete it. To investigate the role of secreted S100A11 in MPM, we inhibited its function by neutralizing S100A11 with an antiS100A11 antibody. Interestingly, the antibody significantly inhibited the proliferation of S100A11-secreting MPM cells in vitro and in vivo. Microarray analysis revealed that several pathways including genes involved in cell proliferation were negatively enriched in the antibody-treated cell lines. In addition, we examined the secretion level of S100A11 in various types of pleural effusions. We found that the secretion of S100A11 was significantly higher in MPM pleural effusions, compared to others, suggesting the possibility for the use of S100A11 as a biomarker. In conclusion, our results indicate that extracellular S100A11 plays important roles in MPM and may be a therapeutic target in S100A11secreting MPM.
\end{abstract}

\section{Introduction}

Malignant pleural mesothelioma (MPM) is a highly invasive and aggressive tumor that develops in the mesothelial lining of the pleura. The median survival of patients with MPM from the time of diagnosis is usually less than 1 year $^{1,2}$. While surgical resection is the treatment of first choice for early-stage disease, recurrence of the disease often makes the prognosis poorer. In addition, most MPM cases are of advanced-stage disease, for which

\footnotetext{
Correspondence: Shinichi Toyooka (toyooka@md.okayama-u.ac.jp) 'Department of General Thoracic Surgery and Breast and Endocrinological Surgery, Okayama University Graduate School of Medicine, Dentistry and Pharmaceutical Sciences, Okayama, Japan

${ }^{2}$ Department of Cell Biology, Okayama University Graduate School of Medicine, Dentistry and Pharmaceutical Sciences, Okayama, Japan

Full list of author information is available at the end of the article
}

the benefits of a standard chemotherapeutic regimen with cisplatin and pemetrexed are very limited. These considerations demand the development of novel therapeutic strategies for MPM.

Proteins of the S100 family are small molecules (ranging from 9 to $14 \mathrm{kDa}$ ) with two EF-hands and in humans, the family is composed of 20 different members (S100A1-S100A16, S100ק, S100G, S100P, and S100Z). This group of proteins modulates a variety of cellular processes, including cell proliferation, differentiation, and intracellular signaling by functioning both as intracellular $\mathrm{Ca}^{2+}$ sensors and as extracellular factors ${ }^{3-5}$.

S100A11, also called S100C or calgizzarin, was cloned from chicken gizzard in $1991^{6}$. We previously reported that S100A11 has two ambivalent functions in the cells. 
Namely, in the cytoplasmic compartment, S100A11 inhibits the growth of normal human keratinocytes in response to high $\mathrm{Ca}^{2+}$ or transforming growth factor $\beta^{7,8}$. Contrarily, the binding of extracellular S100A11 to the receptor for advanced glycation end products (RAGE) enhances the production of epidermal growth factor family proteins, resulting in growth stimulation ${ }^{5,9}$. Based on these findings, we have studied the biological activity of S100A11 by focusing both on intracellular and extracellular S100A11. As for the function of intracellular S100A11, we have shown that the intracellular S100A11-ANXA2 complex helps plasma membrane repair, which was critical for survival and metastasis, in metastatic breast cancer cell line ${ }^{10}$. Additionally, it is reported that intracellular S100A11 promotes pseudopodial actin dynamics, which plays a critical role in tumor metastasis and the suppression of S100A11 results in inhibition of cell migration and invasion, and the reversion of Epithelial to mesenchymal transition (EMT) in various metastatic cell lines ${ }^{11}$. Regarding extracellular S100A11, we have recently reported that, in mesothelioma cells, S100A11 dimerizes in the peroxisome after transportation of monomeric S100A11 through the interaction with PEX14, an essential component of peroxisomal import machinery, and actively secreted ${ }^{12}$. However, despite advances in the understanding of the biological activity and mechanisms of this protein, little is known about its therapeutic or diagnostic potential. In this study, we investigated the relationship between extracellular S100A11 and MPM, and explored the possibility of an intervention in S100A11 function for MPM treatment and diagnosis.

\section{Results}

\section{Secretion levels of S100A11 in malignant cell lines and} overexpression of S100A11 in MPM

We first examined the secretion level of S100A11 in the culture media of various cell lines by enzyme-linked immunosorbent assay (ELISA). Seven MPM, 2 normal mesothelial, 12 lung cancer, 3 gastric cancer, 3 colorectal cancer, and 3 breast cancer cell lines were used for this analysis, and the result is shown in Fig. 1a. We detected increased levels of S100A11 in cancer cells with various secretion levels. Of interest, there was the marked difference in S100A11 secretion between MPM cells and normal cells. All examined MPM cell lines except for MSTO-211H commonly secreted S100A11, whereas no secretion was observed in normal mesothelial cell lines. MPM cell lines were classified into three categories based on the secretion level of S100A11: High (YUMC44, H290, and H28), Low (HP-1, H2452, and H2052), and None (MSTO-211H). To investigate the correlation between S100A11 secretion and protein expression, protein expression levels of S100A11 in MPM and normal mesothelial cell lines were determined by western blot analysis. S100A11 was significantly overexpressed in MPM cell lines, compared to normal mesothelial cell lines (Fig. 1b). To confirm the same phenomenon in clinical MPM specimens, three histological subtypes of MPM tissues, epithelioid, sarcomatoid, and biphasic type, were obtained from the patients who underwent surgery at the Okayama University Hospital and then studied for S100A11 expression. We also prepared the paraffin blocks filled with $\mathrm{H} 2452$ and MeT-5A cells to use as a positive and negative control, respectively. Immunohistochemistry demonstrated that S100A11 was mainly localized in the cytoplasm or nucleus and was strongly positive in MPM cells, but not in surrounding normal lung cells. Representative images are shown in Fig. 1c. Taken together, these results suggest that S100A11 is aberrantly overexpressed in MPM at both cultured cells and clinical samples and secreted from MPM cells.

\section{Inhibition of the amount of extracellular S100A11 in MPM cells}

Next, we examined the involvement of extracellular S100A11 in the growth regulation of MPM cells. As shown in Fig. 2a, MTT assay revealed that administration of the purified S100A11 recombinant protein to the cultures promoted cellular proliferation of MPM cell lines (H2452 and H2052) in a dose-dependent manner up to the point of $100 \mathrm{ng} / \mathrm{ml}$. The growth induction stimulated by extracellular foreign S100A11 at the concentration of $1000 \mathrm{ng} / \mathrm{ml}$ was almost equal in H2452 cells or tended to little bit decline in $\mathrm{H} 2052$ cells when compared to those at the concentration of $100 \mathrm{ng} / \mathrm{ml}$. While the highest concentration $(10,000 \mathrm{ng} / \mathrm{ml})$ commonly worked as growth inhibition in the both cell lines. These results provided us an optimal concentration of extracellular S100A11 (100 $\mathrm{ng} / \mathrm{ml}$ ) in growth stimulation of MPM cells. To inhibit the function of secreted S100A11, we used an anti-S100A11 antibody (Proteintech Group), which has been previously reported as a neutralizing antibody for S100A11 ${ }^{13}$. Similarly, we confirmed that the screened antibody decreased the quantity of S100A11 in the cell culture media (Fig. $2 b)$. The effect was continued for about $48 \mathrm{~h}$ and then expired at later time point, probably due to the consumption of the antibody life. We also confirmed that moues control IgG had no effect on cell growth of MPM cell lines (H2052 and H2452, data not shown). Based on these results, the anti-S100A11 antibody was administered on day 1 and day 3 in culture system. We found that cellular growth of "Low" cells (H2452 and H2052) was both highly suppressed by an anti-S100A11 antibody with a final concentration of $100 \mathrm{ng} / \mathrm{ml}$ in culture media (Fig. 2c, top). On the other hand, in "High" cells (H290 and $\mathrm{H} 28$ ), the same concentration of the anti-S100A11 antibody did not show any appreciable effect on their cell 


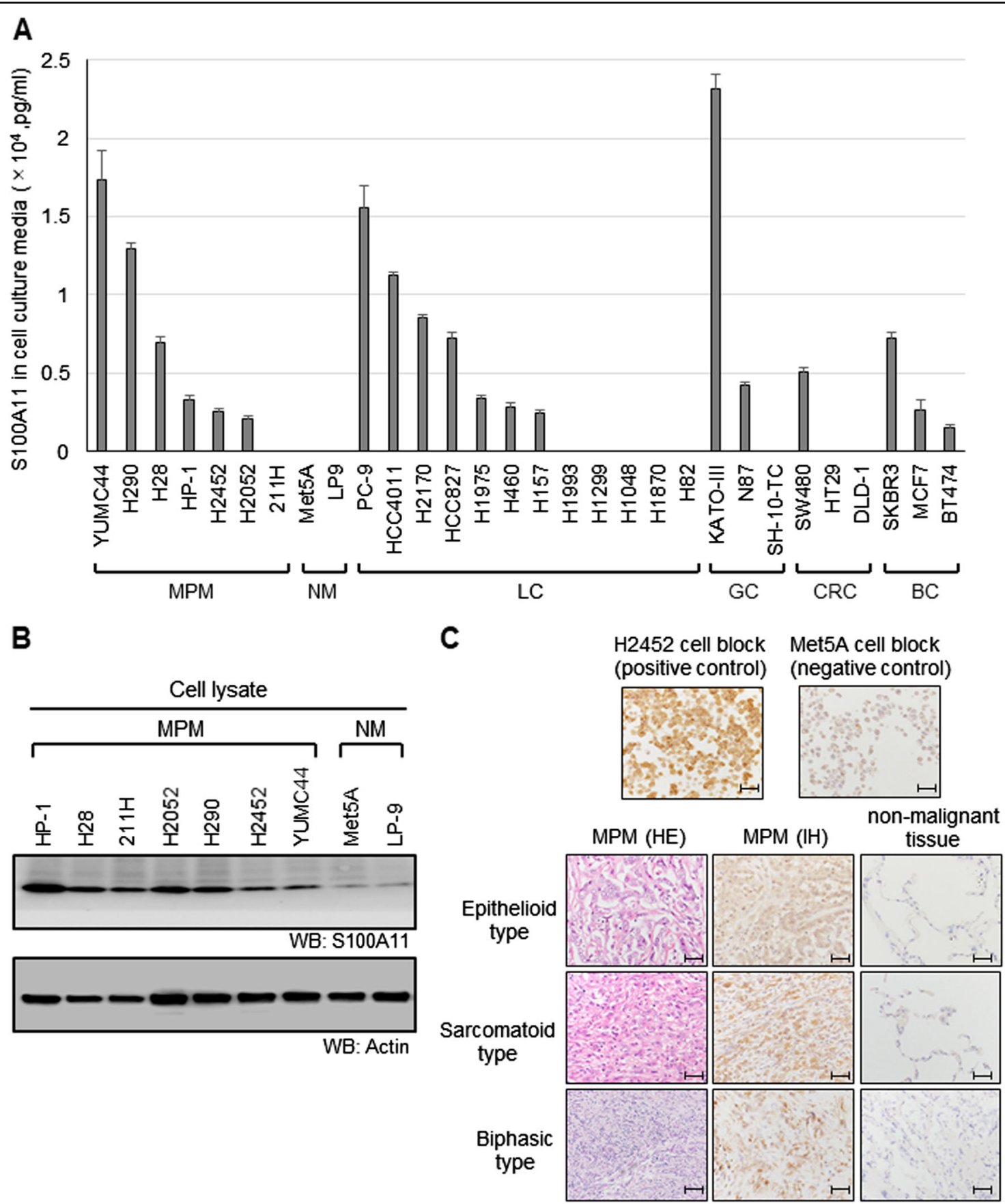

Fig. 1 High secretion and overexpression of S100A11 in MPM. a Concentration of secreted S100A11 in the culture media of various cell lines, as determined by ELISA. The secretion level of S100A11 in all MPM cell lines, except for $211 \mathrm{H}$, is higher than that of NM. 211 H MSTO-211H, NM normal mesothelial cell, LC lung cancer, GC gastric cancer, CRC colorectal cancer, BC breast cancer. b Protein level of S100A11 was higher in MPM cell lines, compared to NM. NM normal mesothelial cell. c Immunohistochemical analysis of S100A11 in surgically resected tissues from patients with MPM. The representative images of three MPM subtypes are shown. The pictures on the left show hematoxylin-eosin (HE)-stained images corresponding to the S100A11-stained images in the middle. Scale bars, $50 \mu \mathrm{m}$ (positive and negative control) and $100 \mu \mathrm{m}$ (clinical samples)

growth. Facing to the issue, we increased the antibody dose up to $1 \mu \mathrm{g} / \mathrm{ml}$. The higher dose gave suppression of cell growth even in these cell lines (Fig. 2c, bottom). As for "None" cells (MSTO-211H), the antibody did not affect the cell growth, regardless of dose (Fig. 2c). To further evaluate the effect of anti-S100A11 antibody on the migration and invasion of MPM, we performed a Boyden chamber assay. Microscopy images of the Boyden 


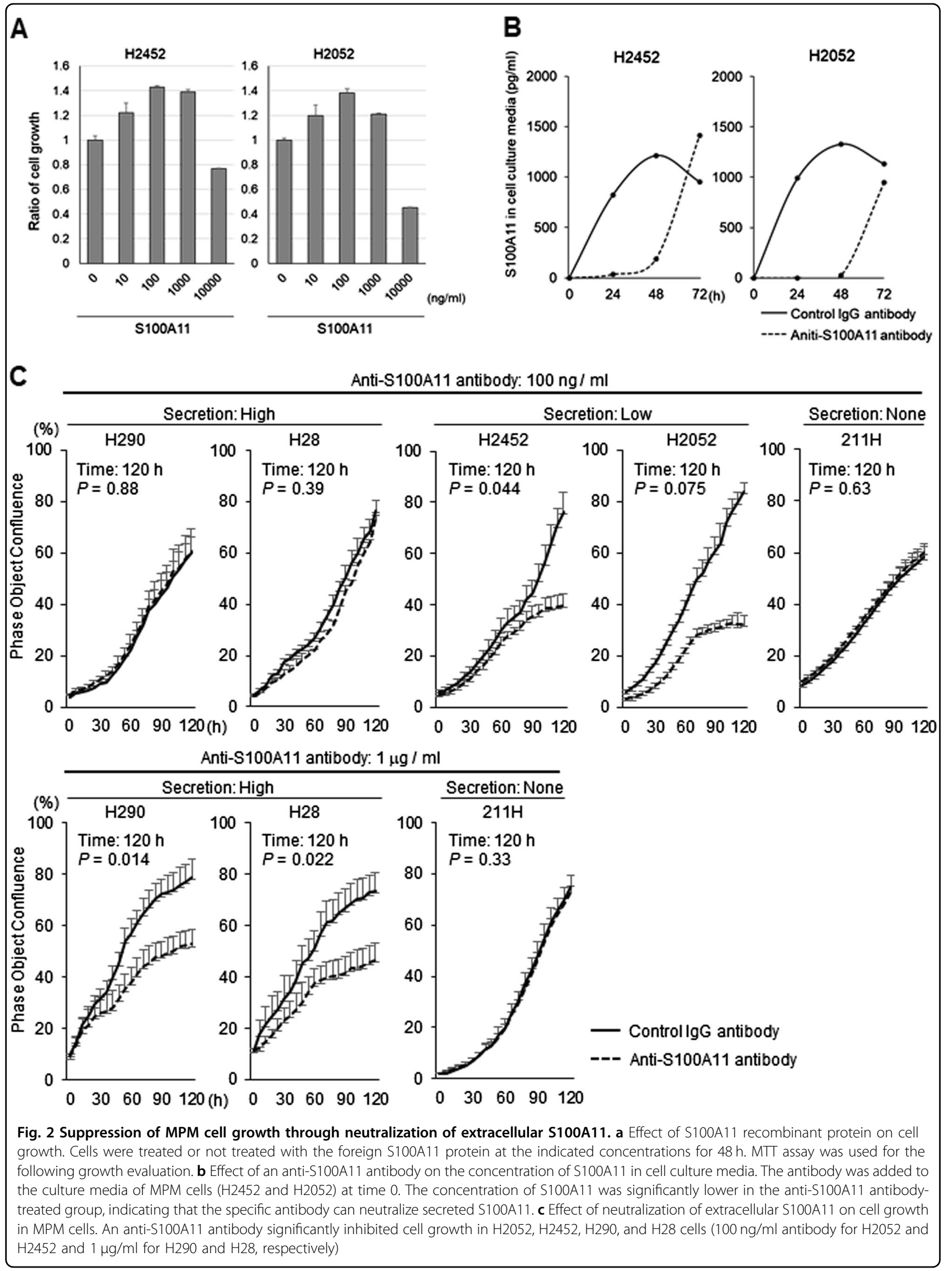


chamber assay are shown in Supplementary Fig. S1A and S1B. Migration and invasion were significantly suppressed in MPM cells treated with the antibody. To confirm the used antibody specificity, we tried to assess whether replenishing foreign S100A11 protein in the culture could cancel the antibody-mediated anticancer behaviors in vitro. As shown in Supplementary Fig. S1A-C, we found that this procedure effectively enfeebled cancer preventive benefits of the antibody in all the three experimental contexts, migration, invasion, and growth of MPM cell lines (H2452 and H2052).

There is a close relationship between cellular growth and cell cycle condition. This prompted us to examine whether cell cycle can be affected by the extracellular S100A11. Cyclins D and B were reliable good makers for cell cycle G1/S-phase and G2/M-phase, respectively, so that we investigated the expression levels of them after stimulation of the cells with $100 \mathrm{ng} / \mathrm{ml}$ of S100A11. By the western blot analysis approach, we found that Cyclin D but not Cyclin B was significantly induced with time dependency after the addition of S100A11 in MPM cultures (H2452 and H2052) (Fig. S1D). In addition, we found that anti-apoptotic Bcl-2 protein was significantly elevated with a similar manner to those of Cyclin D in both $\mathrm{H} 2452$ and H2052 cells (Fig. S1D). These results suggest that the secreted S100A11 positively regulates not only cell cycle, especially in a specific activation of the G1 phase and following G1/S transition through an induction of Cyclin D, but also survival via induction of Bcl-2.

\section{The alteration of downstream signaling affected by the anti-S100A11 antibody and the S100A11 recombinant protein}

To gain insight into the intracellular signaling events induced by the neutralization of extracellular S100A11, we investigated the key molecules regarding to cancer progression. MPM cells were cultured with the antiS100A11 antibody $(1 \mu \mathrm{g} / \mathrm{ml})$, S100A11 recombinant protein $(100 \mathrm{ng} / \mathrm{ml})$, or mouse control IgG $(1 \mu \mathrm{g} / \mathrm{ml})$. Lysates were extracted every $6 \mathrm{~h}$ and subjected to western blot analysis (Supplementary Fig. S2). As a result, we found that the treatment with the anti-S100A11 antibody significantly suppressed the constitutive phosphorylation of endogenous STAT3 in H2452 and H2052 cells. Unexpectedly, in cases of AKT and MAPK, both phosphorylation status were commonly upregulated temporary at once at $6 \mathrm{~h}$ and then tended to be gradually suppressed with a time-dependent manner (Supplementary Fig. S2A). This may be possibly explained by compensation of the STAT3 downregulation. Next as a converse experiment, we stimulated the cells with extra recombinant S100A11 under the absence of the antibody in culture. The addition of S100A11 recombinant protein in turn activated the downstream proteins, STAT3, AKT, and MAPK
(Supplementary Fig. S2B). To further specify the central pathway related to the extracellular S100A11-induced proliferation of MPM cells, we tried to inhibit the intracellular activations of STAT3, MEK, and PI3K and assessed the following cellular proliferations. Unexpectedly, we found that either Stattic: STAT3 inhibitor, Trametinib: MEK inhibitor, or Taselisib: PI3K inhibitor, effectively suppressed the S100A11-stimulated cellular proliferation with a similar inhibitory degree, suggesting that these molecules play a crucial role in cellular growth and they cooperatively function toward same way regarding growth upregulation under the downstream of RAGE upon S100A11 binding (Supplementary Fig. S2D). Taken together, these results strongly suggest that the secreted S100A11 plays a critical role on MPM progression through upregulation of cell growth, motility, and invasion with an autocrine manner and the extracellular S100A11 is becoming a prominent molecule for therapeutic target of the MPM progression.

\section{An anti-S100A11 antibody inhibits the tumorigenic potential in a mouse xenograft model of MPM}

We investigated the antitumor effect of the validated anti-S100A11 neutralizing antibody on the MPM in a xenograft mouse model, using $\mathrm{H} 2452$ and $\mathrm{H} 290$ cells. We subcutaneously injected tumor cells and the anti-S100A11 antibody, and evaluated the effect of the antibody on the tumorigenic potential. As shown in Fig. 3a, b, the tumor growth in the antibody-administered group was significantly suppressed, compared to those of the control group.

\section{Microarray analysis of $\mathrm{H} 2052$ and $\mathrm{H} 2452$ cells treated with an S100A11 neutralizing antibody}

To reveal the mechanisms involved in the anticancer activity induced by neutralization of extracellular S100A11, we carried out gene expression microarray analysis. The details of the expression profile studies are reported in Supplementary Table S1. Clustering analysis based on the transcripts showed a clear distinction between the cell lines treated or untreated with the antibody (Fig. 4). Next, a gene set enrichment analysis (GSEA) was performed to clarify the groups of genes affected by anti-S100A11 antibody administration. We found that several pathways, including genes involved in cell proliferation (HALLMARK_MITOTIC_SPINDLE), (HALLMARK_P53_PATHWAY 1), (HALLMARK_PI3K_AKT_MTOR_SIGNALING), and (HALLMARK_KRAS_SIGNALING_DN), and a subgroup of genes related to protein secretion (HALLMARK_PROTEIN_SECRETION) were negatively enriched in the antibody-treated cell lines (Table 1). These results are consistent with our in vitro and in vivo data. 


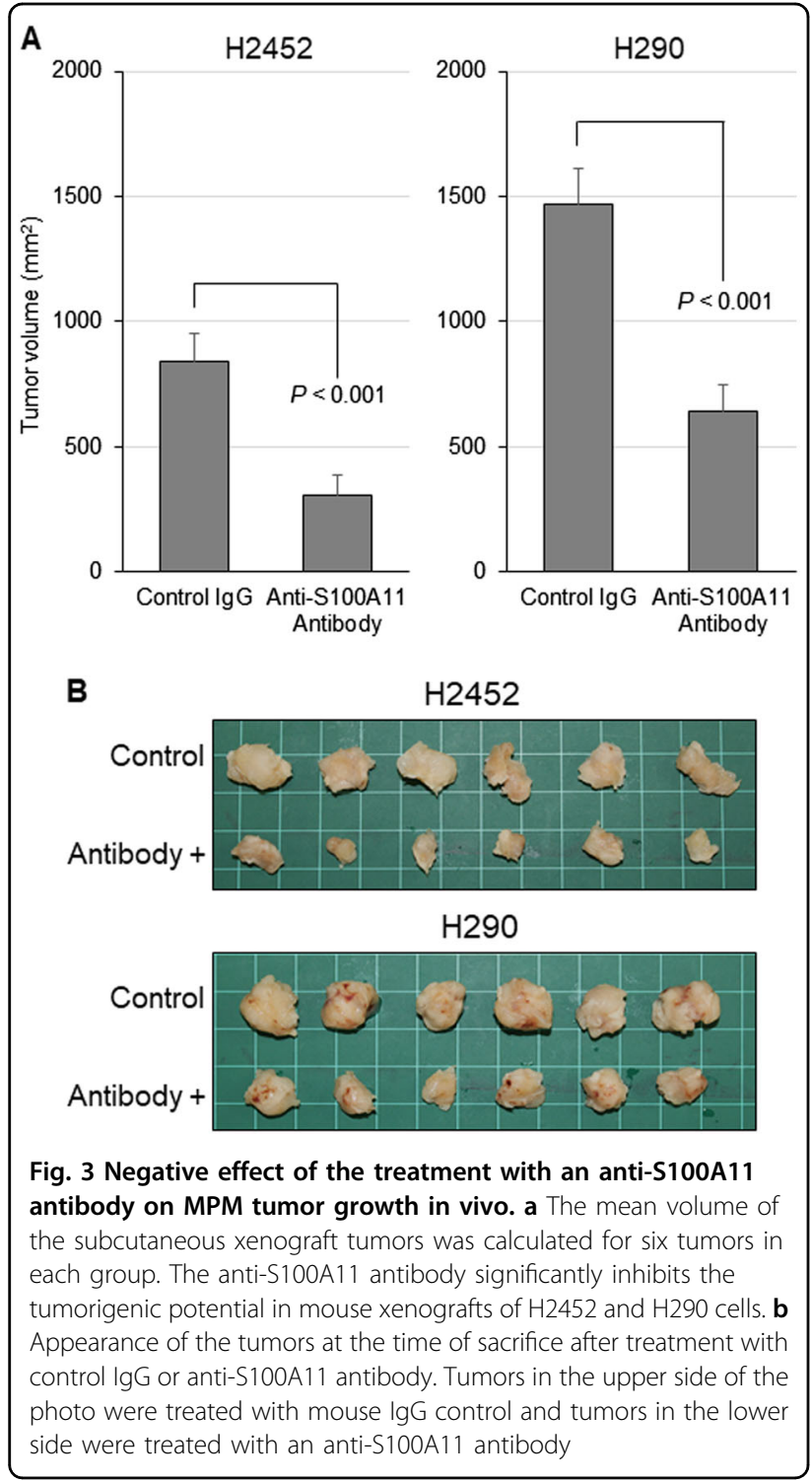

\section{Function of RAGE as a receptor for S100A11}

To explore the function of RAGE as a receptor for S100A11 in MPM, we first confirmed the positive expression of RAGE in MPM cell lines by western blot analysis (Fig. 5a). To prevent the intrinsic RAGE activation caused by ligand binding, we used sRAGE, which acts as decoy to compete with S100A11, the ligand for the cellular RAGE, and examined a series of effector molecules regarding to RAGE downstream signaling. MPM cells were treated or not treated with S100A11 recombinant protein $(100 \mathrm{ng} / \mathrm{ml})$ under the presence or absence of sRAGE $(1 \mu \mathrm{g} / \mathrm{ml})$. Twenty-four hours later, the treatedcell lysates were prepared and then subjected to western blot analysis. We found that blockage of RAGE signaling suppressed the phosphorylation of STAT3 and MAPK induced by S100A11 stimulation (Fig. 5b). Additionally,

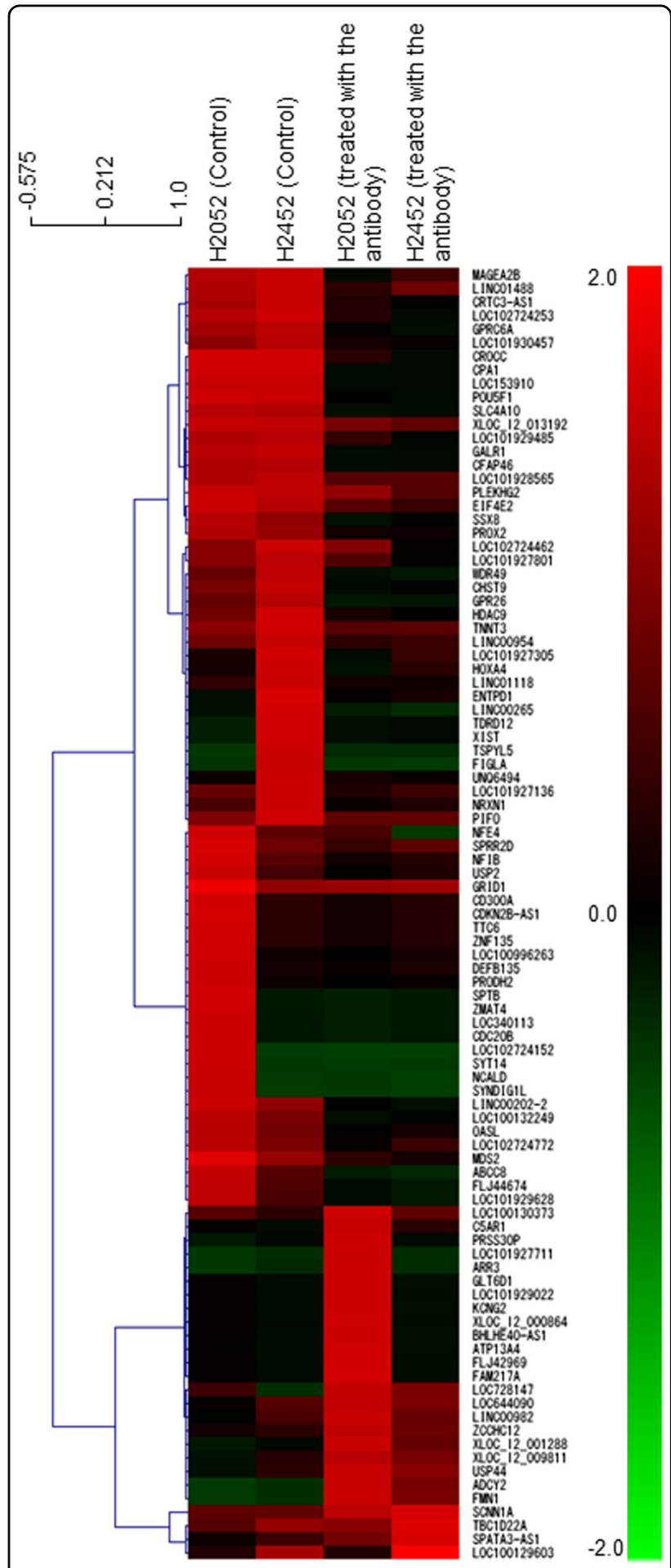

Fig. 4 Global gene expression changes upon treatment with an anti-S100A11 antibody Clustering and heatmap of MPM cell lines are shown. Paired comparison analysis based on 50,739 probes indicated 2222 differentially expressed transcripts between antibodytreated MPM cell lines and parental MPM cell lines (H2052 and H2452). Clustering using Pearson correlation and average linkage was based on these transcripts. The color bar represents the expression values after $\log 2$ transformation 
Table 1 Enriched pathways in the parental and antibodytreated cell lines

\begin{tabular}{lll}
\hline & ES & $\begin{array}{c}\text { FDR } \\
\boldsymbol{q} \text {-val }\end{array}$ \\
\hline GSEA pathway (parental cell line, $n=2)$ & & \\
HALLMARK_PANCREAS_BETA_CELLS & 0.83 & 0.274 \\
HALLMARK_REACTIVE_OXIGEN_SPECIES_PATHWAY & 0.6 & 0.274 \\
HALLMARK_COAGULATION & 0.52 & 1.000 \\
HALLMARK_PEROXISOME & 0.51 & 0.995 \\
HALLMARK_MYC_TARGETS_V2 & 0.44 & 0.926 \\
HALLMARK_GLYCOLYSIS & 0.42 & 0.722 \\
GSEA pathWay (anti-S100A11 antibody-treated cell line, & & \\
n = 2) & & \\
HALLMARK_PI3K_AKT_MTOR_SIGNALING & -0.56 & 0.819 \\
HALLMARK_PROTEIN_SECRETION & -0.47 & 0.47 \\
HALLMARK_KRAS_SIGNALING_DN & -0.47 & 0.811 \\
HALLMARK_MYOGENESIS & -0.47 & 0.87 \\
HALLMARK_MITOTIC_SPINDLE & -0.33 & 0.887 \\
HALLMARK_P53_PATHWAY & -0.35 & 0.409 \\
\hline
\end{tabular}

ES enriched score, FDR false discovery rate

MTT assays revealed that sRAGE mitigated growth promotion in both $\mathrm{H} 2452$ and $\mathrm{H} 2052$ cells, indicating that S100A11-RAGE axis plays a pivotal role in MPM progression in response to the secreted extracellular S100A11 (Fig. 5c).

\section{Secretion levels of S100A11 in pleural effusions}

To explore the possibility of using S100A11 as a useful marker for diagnosis of MPM, we examined the secretion levels of S100A11 in several types of pleural effusions. We obtained pleural effusions from 29 MPM patients (biphasic type: $n=10$, epithelioid type: $n=15$, sarcomatoid type: $n=4), 11$ benign asbestosis (BA) patients, and 12 patients who underwent thoracic surgery at the Okayama University Hospital and the National Hospital Organization Yamaguchi-Ube Medical Center (Ube, Yamaguchi, Japan). Postoperative pleural effusions were obtained from lung cancer $(n=10)$ or pulmonary cyst $(n$ $=2$ ) patients after postoperative day 2. The concentration of S100A11 was measured by ELISA. As shown in Fig. 6, the level of S100A11 was remarkably elevated in pleural effusion obtained from MPM patients when compared to that obtained from BA patients as a benchmark $(P=$ 0.014). Furthermore, we found that the secretion level of S100A11 was significantly higher in pleural effusion from BA patients than that from postoperative patients $(P=$ 0.041).

\section{Discussion}

In this study, we found that extracellular S100A11, via RAGE, has a critical role in tumor progression of MPM. The blockage of extracellular S100A11 with a neutralizing antibody inhibited the cell proliferation, migration, and invasion of MPM cells. Additionally, in a mouse xenograft model, tumorigenesis of MPM cells was markedly inhibited by an anti-S100A11 antibody. These results suggest that extracellular S100A11 is a potential therapeutic target for MPM. Interestingly, we found that protein expression levels of S100A11 are not correlated with secretion levels in various cells lines, suggesting that intracellular increasing in stock level of S100A11 protein is not essential factor for its secretory mechanism. In fact, MSTO-211H showed no secretion of S100A11 even though plenty of S100A11 protein was stored in cells. To clarify the reason of this discrepancy at the molecular level, further comprehensive investigation with focusing on secretory pathways of leaderless proteins is indispensable.

One of the interesting features of S100A11 is to possess different functions depending on its location and on different cell types, as we reported previously ${ }^{9}$. Accumulating evidence indicates that S100A11 expression is upregulated in various cancers and promote cancer development ${ }^{14-17}$. However, this is not common in all cancer species, for example, low expression of S100A11 in bladder cancers is associated with poor survival in the patients ${ }^{18}$. Therefore, S100A11 might act sometimes as an oncogene, and sometimes as a suppressor gene, and have a key role in the progression of malignant tumors based on the balance of its ambivalent effect, which may be different depending on cancer types. When considering the use of this protein for treatment, the targeting of intracellular S100A11 would require the unitarily control of its two different functions. Thus, it is more convenient to focus on extracellular S100A11 for developing new therapeutic strategies for MPM.

RAGE, which was first identified as a RAGE, is known to bind different ligands, such as amyloid-beta peptide, HMGB1 (amphoterin), and some members of the S100 family, including S100A11 ${ }^{19-21}$. We found that RAGEpositive MPM cells constitutively express and secrete S100A11, the S100A11-RAGE axis is greatly involved in sustained phosphorylation of downstream effector molecules, STAT3, MAPK, and PI3K-AKT, and the blockage of S100A11-RAGE connection using either sRAGE or S100A11 neutralizing antibody effectively inhibits cell growth of MPM cells. For the S100A11-mediated growth upregulation, Cyclin D and $\mathrm{Bcl}-2$ might be critically relevant. How are these molecules induced under the RAGE activation? One hint may come from the Fig. S2D. The S100A11-induced Cyclin D but not Bcl-2 was significantly downregulated by the STAT3, MEK and PI3K 

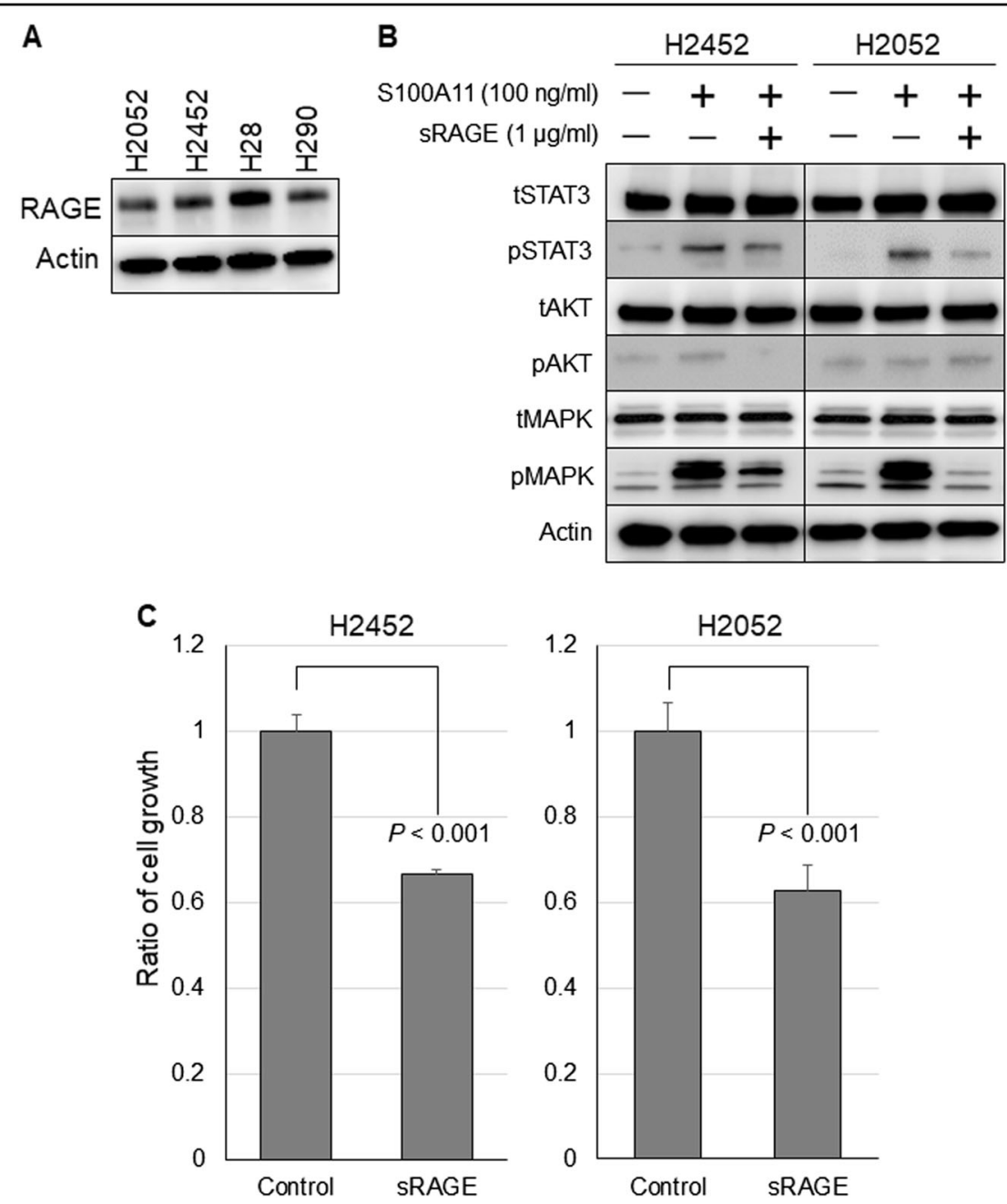

Fig. 5 Secreted S100A11 exerts its important role in the proliferation of MPM cells via RAGE. a RAGE protein expression in MPM cells. $\mathbf{b}$ Inhibition of RAGE signaling through sRAGE significantly suppressed the phosphorylation of STAT3 and MAPK induced by S100A11 recombinant protein. $\mathbf{c}$ The effect of SRAGE on the growth of MPM cells examined by MTT assay. Cell proliferation was significantly inhibited by sRAGE

inhibitors, suggesting that the molecules regarding pathways coordinately regulate Cyclin D expression after the RAGE activation upon S100A11 binding. Through this study, we hence strongly recognized a significant role of secreted S100A11 in mesothelioma progression.

In our experiments, growth inhibitory effect of sRAGE was limited and insufficient in comparison with the that of anti-S100A11 antibody, indicating that an additional S100A11 receptor, such as CD36, might activate survival or proliferative pathways in the cells ${ }^{9,22}$. Moreover, RAGE has been shown to have many distinct biological functions. For instance, van Zoelen and colleagues ${ }^{23}$ demonstrated that RAGE signaling contributed to an effective antibacterial defense during Escherichia coli infection (inhibition of bacterial outgrowth and dissemination), and RAGE deficiency resulted in enhanced organ injuries due to liver necrosis. Thus, the adverse effects due to the inhibition of RAGE signaling are still unclear and as a therapeutic target for MPM, it seems more convenient to inhibit extracellular S100A11 than RAGE at present.

Although "High" cells (H290 and H28) displayed resistance to the S100A11 antibody at lower concentration $(100 \mathrm{ng} / \mathrm{ml})$, the effect of S100A11 antibody was shown when much higher concentration of it $(1 \mu \mathrm{g} / \mathrm{ml})$ was used. Thus, the secretion of S100A11 in MPM cells was prerequisite for the antibody approach and measurement of the secretion level of S100A11 was quite important index to predict an effectiveness of the S100A11 antibody. We hence examined the secretion level of S100A11 in pleural effusion. The concentration of S100A11 in MPM pleural effusions was significantly higher than that in pleural effusions of BA and postoperative patients, though the amount of secreted S100A11 is high in pleural effusions from both patients with MPM and BA. Generally, BA is 


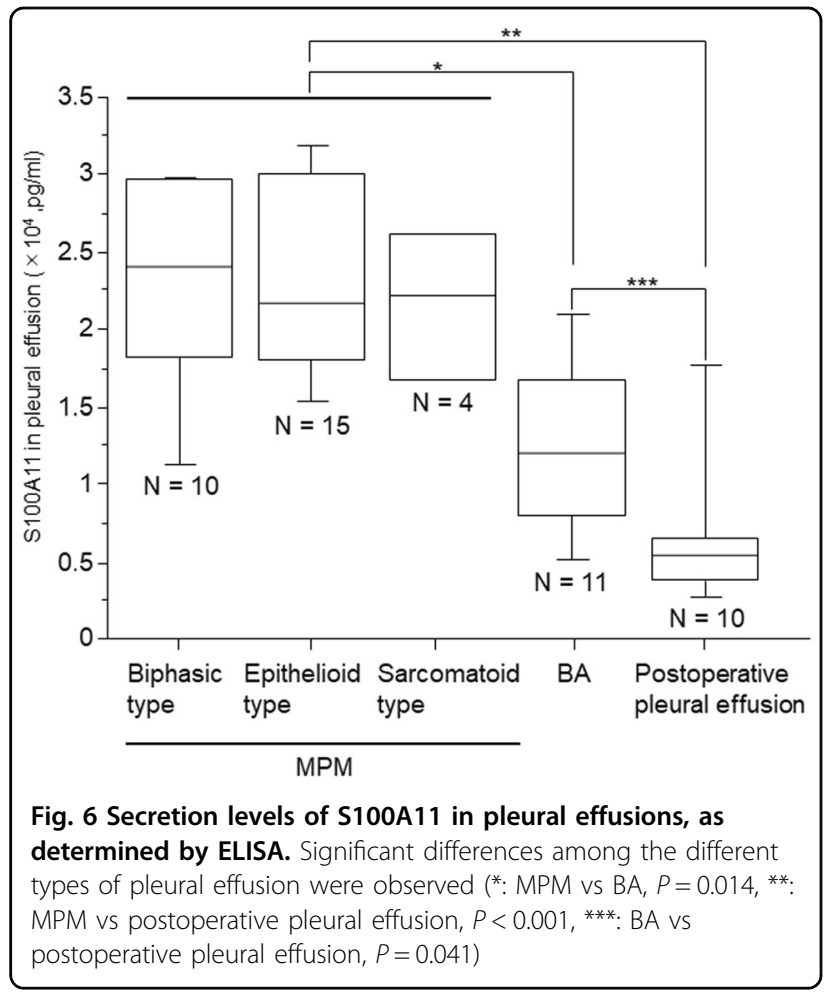

not regarded as a precancerous lesion of mesothelioma. Taking it into account, we consider that MPM cells actively secrete S100A11 in pleural effusion. These results suggest that S100A11 can be not only a therapeutic target but also an early diagnosis marker. In addition, it is of note mentioning that the concentration of S100A11 in the pleural effusions of patients with BA was significantly higher than that of postoperative patients. The association between high expression of proteins of the S100 protein family and inflammatory diseases has been reported ${ }^{24-27}$; this along with our results suggest that S100A11 may be involved in chronic inflammation.

In conclusion, we showed that extracellular S100A11 plays an important role in MPM progression and neutralization of extracellular S100A11 directly linked to marked suppression of MPM developments in vitro and in vivo. Our results suggest that extracellular S100A11 can be a novel, selective and effective therapeutic target in S100A11-secreting MPM, since normal cells displayed no secretion phenotype of S100A11.

\section{Materials and methods \\ Cell lines and reagents}

We used seven human MPM cell lines [NCI-H28 (H28), NCI-H290 (H290), NCI-H2052 (H2052), NCI-H2452 (H2452), HP-1, YUMC44, and MSTO-211H], two human normal mesothelial cell lines (MeT-5A and LP-9), 10 human lung cancer cell lines [HCC827, PC-9, HCC4011, NCI-H1975 (H1975), NCI-H1993 (H1993),
NCI-H1299 (H1299), NCI-H460 (H460), NCI-H2170 (H2170), NCI-H157 (H157), and NCI-H82 (H82)], two human extrapulmonary small-cell cancer cell lines [NCIH1048 (H1048) and NCI-H1870 (H1870)], three gastric cancer cell lines [KATO III, NCI-N87 (N87), and SH-10TC], three colorectal cancer cell lines (SW480, HT29, and DLD-1), and three breast cancer cell lines (MCF-7, BT474, and SK-BR-3). Two MPM cell lines (HP-1 and H2452) were established by one of the authors (H.I.P.) ${ }^{28}$. Three MPM cell lines (H28, H290, and H2052 obtained in 2002), two lung cancer cell lines (HCC4011 and H82 obtained in 2008), and two extrapulmonary small-cell cancer cell lines (H1048 and H1870 obtained in 2008) were kindly gifted by Dr. Adi F. Gazdar (Hamon Center for Therapeutic Oncology Research and Department of Pathology, University of Texas Southwestern Medical Center at Dallas, Dallas, TX, USA) ${ }^{29}$. YUMC44 cell line was established by one of the authors (H.Y.) ${ }^{30}$. MSTO211H, MeT-5A, N87 cell lines, seven lung cancer cell lines (HCC827, H1975, H1993, H1299, H460, H2170, and H157), three breast cancer cell lines (MCF-7, BT474, and SK-BR-3), and three colorectal cancer cell lines (Sw480, HT29, and DLD-1) were purchased from the American Type Culture Collection (Manassas, VA, USA). Two gastric cancer cell lines (KATO III and SH-10-TC) were obtained from the Cell Resource Center for Biomedical Research Institute of Development Aging and Cancer, Tohoku University (Sendai, Miyagi, Japan). LP-9 cell line was purchased from the Coriell Cell Repository (Camden, NJ, USA). PC-9 cell line was purchased from Riken Cell Bank (Tsukuba, Ibaragi, Japan). The cell lines within 30 passages were used in this study and the cumulative culture length was less than 6 months. For cell lines with long-term preservation in liquid nitrogen, DNA fingerprinting analysis by short tandem repeat profiling (the PowerPlex 1.2 System, Promega) was performed for the cell authentication. All the cell lines, except for LP-9 and breast cancer cell lines, were maintained in RPMI-1640 medium (Sigma-Aldrich, St Louis, MO, USA, Product No. R8758) supplemented with $10 \%$ fetal bovine serum (FBS). The three breast cancer cell lines were cultured in Dulbecco's modified Eagle's medium (Sigma-Aldrich, Product No. D6429) with 10\% FBS. LP-9 was cultured in Ham's F12 medium (Sigma-Aldrich, Product No. N4888)/Medium 199 (Sigma-Aldrich, Product No. M7653) (1:1 mixture) with 10\% FBS, 2 mM L-glutamine (Thermo Fisher Scientific, Waltham, MA, USA, catalog \#25030-081), $1.7 \mathrm{nM}$ epidermal growth factor (Sigma-Aldrich, Product No. E9644), and $1100 \mathrm{nM}$ hydrocortisone (Sigma-Aldrich, Product No. H0888). All the cell lines were grown in a humidified incubator with $5 \% \mathrm{CO}_{2}$ at $37^{\circ} \mathrm{C}$ and routinely tested for mycoplasma by Venor GeM OneStep kit (Minerva Biolabs, Berlin, Germany, Product No. 11-8050). Soluble receptor for 
advanced glycation end products (RAGE) Fc chimera (sRAGE) (R\&D Systems, Minneapolis, MN, USA, accession \#Q15109), static (Abcam, Cambridge, MA, USA, ab120952), trametinib (GSK-1120212) (LC Laboratories, Woburn, MA, USA, catalog \#T-8132), Taselisib (GDC0032) (Selleckchem, Houston, TX, USA, catalog \#S7103), and the mouse IgG-isotype control (Abcam, ab37355) were obtained from the designated sources. S100A11 recombinant protein was prepared as described previously ${ }^{5}$.

Determination of the concentration of S100A11 by ELISA

The concentration of S100A11 was measured using the CircuLex S100A11 ELISA Kit (Circulex, Nagano, Japan, Code No. CY-8063) according to the manufacturer's protocol. The standard curve for the ELISA was obtained using various concentrations of recombinant human S100A11. Samples were diluted 5- to 10-fold. The absorbance was measured at the dual wavelengths of 450/ $540 \mathrm{~nm}$ using the Flex Station 3 microplate reader (Sunnyvale, CA, USA) and the concentration of S100A11 (in $\mathrm{pg} / \mathrm{ml}$ ) was calculated according to the standard curve.

\section{Western blot analysis}

The detailed protocol has been described previously ${ }^{31}$. The primary antibodies used for western blot analyses were as follows: EGFR (catalog \#4267), phospho- (p-) EGFR (Tyr1068) (\#3777), Stat3 (\#12640), p-Stat3 (Tyr705) (\#9145), Akt (\#9272), p-Akt (Ser473) (\#4060), p44/p42 MAPK (\#9102), p-p44 / p42 MAPK (\#9101), Cyclin B1 (\#4138), Cyclin D1 (\#2922), Bcl-2 (\#2872) (Cell Signaling Technology, Danvers, MA, USA), S100A11 (Medical \& Biological Laboratories, Nagoya, Japan, catalog \#CYM1037), and $\beta$-actin (used as a loading control) (Merck Millipore, Billerica, MA, USA, catalog \# MAB1501). The following secondary antibodies were used: goat anti-rabbit (catalog \#sc-2030) or anti-mouse (catalog \#sc-2031) immunoglobulin G (IgG)-conjugated horseradish peroxidase (Santa Cruz Biotechnology, Dallas, TX, USA). To detect specific signals, the membranes were examined using the ECL Prime Western Blotting Detection System (GE Healthcare, Amersham, UK, Code No. RPN2235) and LAS-3000 imager (Fujifilm, Tokyo, Japan).

\section{Immunohistochemical analysis of clinical samples}

MPM tissues were obtained from patients who underwent surgery at the Okayama University Hospital, in Okayama City, Japan. The experimental protocol was approved by the Institutional Review Board/Ethical Committee of the Okayama University Graduate School of Medicine, Dentistry and Pharmaceutical Sciences and Okayama University Hospital (Permit Number: 1508-027) and informed consent, including publication of patient photos, was obtained from all the patients. Tissue samples were fixed in $10 \%$ formaldehyde and embedded in paraffin. The immunohistochemical (IHC) staining for S100A11 was performed using an S100A11 primary antibody (R\&D Systems, accession \#P31949). The detailed protocol for the IHC staining has been described previously $^{32}$.

\section{Cell proliferation assays}

To examine the effect of the anti-S100A11 antibody (Proteintech Group, Chicago, IL, USA, catalog \#10237-1AP) on cell proliferation, the IncuCyte Zoom Live Cell Imaging System (Essen Biosciences, Ann Arbor, Michigan, USA) was used. In brief, $1.0 \times 10^{4}$ cells were seeded into each well of a 24-well plate. After $24 \mathrm{~h}$ of incubation, the plates were placed in the Incucyte Zoom system. Images were taken every $6 \mathrm{~h}$ for the indicated amount of time. The percent of confluence over time was calculated using the Incucyte Zoom software. For experiments testing the effect of sRAGE on cell proliferation, the thiazolyl blue tetrazolium bromide (MTT) (Sigma-Aldrich, catalog \#M2128) dye reduction method was used, as described previously ${ }^{33}$.

\section{Cell migration and invasion assays}

Cell migration and invasion were analyzed using a Boyden chamber assay. The cells were cultured with an antibody (100 $\mathrm{ng} / \mathrm{ml}$ ) for $24-48 \mathrm{~h}$ (for migration assays) or $48-72 \mathrm{~h}$ (for invasion assays). The detailed protocol has been described previously ${ }^{34}$.

\section{Xenograft model}

The protocol was approved by the Animal Care and Use Committee of Okayama University (Permit Number: OKU-2016205). Six-week-old NOD/SCID female mice were purchased from Charles River Laboratories (Yokohama, Kanagawa, Japan). To evaluate the effect of the anti-S100A11 neutralizing antibody, mice were randomly divided into two groups: an antibody-administered group and a control group ( $n=3$ for each group). Each cell line $\left(5 \times 10^{6}\right.$ cells $)$ suspended in $200 \mu \mathrm{L}$ RPMI-1640 media and Matrigel Basement Membrane Matrix (Corning, NY, USA, catalog \#354234) was subcutaneously injected into the backs of the mice either with $1 \mu \mathrm{g} / \mathrm{ml}$ anti-S100A11 antibody (proteintech Group) or mouse IgG-isotype control (two tumors per mouse). The injection was repeated five times every 3 days. Four weeks after the first injection, the mice were sacrificed and the tumors were harvested, measured, and photographed. The tumor volume was calculated using the empirical formula $V=1$ / $2 \times[\text { (shortest diameter })^{2} \times($ the longest diameter $\left.)\right]$. No randomization and no blinding was performed. Sample size estimates were based on our previous experience. 


\section{Microarray and GSEA}

Before and after treatment of H2052 and H2452 cells with the anti-S100A11 antibody (Proteintech Group), total RNAs were extracted from cell lines using an RNeasy Mini Kit (Qiagen, Venlo, the Netherlands, catalog \#74104). Purified total RNA samples were hybridized on the Human Whole Genome DNA Microarray system (SurePrint G3 Human 8x60K ver. 2.0, Agilent Technologies, Santa Clara, CA) to obtain the altered gene expression profile. The fold change in the expression of individual genes was calculated and genes with fold changes exceeding 2 -fold or below 2-fold were considered up- and downregulated, respectively (Supplementary Table S1). The specific enrichment of gene sets was further analyzed using the GSEA software (GSEA ver. 2.0) downloaded from the GSEA Website (http://software. broadinstitute.org/gsea/index.jsp).

\section{Statistical analyses}

All in vitro experiments were performed at least three times. Data are expressed as the mean \pm standard deviation. All data were analyzed using the JMP 9.0.0 software for Windows (SAS Institute, Inc., Cary, NC, USA). The Student's $t$-tests was used to compare means of continuous scores between two independent groups. Otherwise, the Mann-Whitney $U$-test was used. An $F$-test to compare variances was performed, if necessary. All statistical tests were two-sided, and probability values less than 0.05 indicated statistically significant differences.

\section{Acknowledgements}

The authors thank Dr. Takehiro Matsubara (Biobank, Okayama University Graduate School of Medicine, Dentistry and Pharmaceutical Sciences, Okayama, Japan) and Ms. Fumiko Isobe (Department of General Thoracic Surgery and Breast and Endocrinological Surgery, Okayama University Graduate School of Medicine, Dentistry and Pharmaceutical Sciences, Okayama, Japan) for their technical supports. This study was supported by a Management Expenses Grants.

\section{Author details}

'Department of General Thoracic Surgery and Breast and Endocrinological Surgery, Okayama University Graduate School of Medicine, Dentistry and Pharmaceutical Sciences, Okayama, Japan. ${ }^{2}$ Department of Cell Biology, Okayama University Graduate School of Medicine, Dentistry and Pharmaceutical Sciences, Okayama, Japan. ${ }^{3}$ Department of Bioinformatics, Okayama University Graduate School of Medicine, Dentistry and Pharmaceutical Sciences, Okayama, Japan. ${ }^{4}$ Department of Medical Oncology, National Hospital Organization Yamaguchi-Ube Medical Center, Ube, Yamaguchi, Japan. ${ }^{5}$ Department of Clinical Research, National Hospital Organization Yamaguchi-Ube Medical Center, Ube, Yamaguchi, Japan. ${ }^{6}$ Department of Clinical Genomic Medicine, Okayama University Graduate School of Medicine, Dentistry and Pharmaceutical Sciences, Okayama, Japan. ${ }^{7}$ Department of Thoracic Surgery, National Hospital Organization YamaguchiUbe Medical Center, Ube, Yamaguchi, Japan. ${ }^{8}$ Department of Cardiothoracic Surgery, New York University Langone Medical Center, New York, NY, USA

\section{Publisher's note}

Springer Nature remains neutral with regard to jurisdictional claims in published maps and institutional affiliations.

Supplementary Information: The online version of this article (https://doi. org/10.1038/s41389-017-0017-3) contains supplementary material.

Received: 27 June 2017 Accepted: 21 September 2017

Published online: 24 January 2018

\section{References}

1. Milano, M. T. \& Zhang, H. Malignant pleural mesothelioma: a population-based study of survival. J. Thorac. Oncol. 5, 1841-1848 (2010).

2. Robinson, B. W. \& Lake, R. A. Advances in malignant mesothelioma. N. Engl. J. Med. 353, 1591-1603 (2005).

3. Bresnick, A. R., Weber, D. J. \& Zimmer, D. B. S100 proteins in cancer. Nat. Rev. Cancer 15, 96-109 (2015).

4. Donato, R. S100: a multigenic family of calcium-modulated proteins of the EFhand type with intracellular and extracellular functional roles. Int. J. Biochem Cell. Biol. 33, 637-668 (2001).

5. Sakaguchi, M. et al. S100A11, an dual mediator for growth regulation of human keratinocytes. Mol. Biol. Cell. 19, 78-85 (2008).

6. Todoroki, H., Kobayashi, R., Watanabe, M., Minami, H. \& Hidaka, H. Purification, characterization, and partial sequence analysis of a newly identified EF-hand type $13-\mathrm{kDa} \mathrm{Ca}(2+)$-binding protein from smooth muscle and non-muscle tissues. J. Biol. Chem. 266, 18668-18673 (1991).

7. Sakaguchi, M. et al. S100C/A11 is a key mediator of $\mathrm{Ca}(2+)$-induced growth inhibition of human epidermal keratinocytes. J. Cell. Biol. 163, 825-835 (2003).

8. Sakaguchi, M. et al. Truncation of annexin A1 is a regulatory lever for linking epidermal growth factor signaling with cytosolic phospholipase A2 in normal and malignant squamous epithelial cells. J. Biol. Chem. 282, 35679-35686 (2007).

9. Sakaguchi, M. \& Huh, N. H. S100A11, a dual growth regulator of epidermal keratinocytes. Amino Acids 41, 797-807 (2011).

10. Jaiswal, J. K. et al. S100A11 is required for efficient plasma membrane repair and survival of invasive cancer cells. Nat. Commun. 5, 3795 (2014).

11. Shankar, J. et al. Pseudopodial actin dynamics control epithelial-mesenchymal transition in metastatic cancer cells. Cancer Res. 70, 3780-3790 (2010).

12. Saho, S. et al. Active secretion of dimerized S100A11 induced by the peroxisome in mesothelioma cells. Cancer Microenviron. 9, 93-105 (2016).

13. Fan, C. et al. S100A11 mediates hypoxia-induced mitogenic factor (HIMF)induced smooth muscle cell migration, vesicular exocytosis, and nuclear activation. Mol. Cell. Proteom. 10(M110), 000901 (2011).

14. Anania, M. C. et al. S100A11 overexpression contributes to the malignant phenotype of papillary thyroid carcinoma. J. Clin. Endocrinol. Metab. 98, E1591-E1600 (2013).

15. Hao, J. et al. Selective expression of S100A11 in lung cancer and its role in regulating proliferation of adenocarcinomas cells. Mol. Cell. Biochem. 359, 323-332 (2012).

16. Ohuchida, K. et al. S100A11, a putative tumor suppressor gene, is overexpressed in pancreatic carcinogenesis. Clin. Cancer Res. 12, 5417-5422 (2006).

17. Oue, N. et al. Gene expression profile of gastric carcinoma: identification of genes and tags potentially involved in invasion, metastasis, and carcinogenesis by serial analysis of gene expression. Cancer Res. 64, 2397-2405 (2004).

18. Memon, A. A. et al. Down-regulation of $\mathrm{S} 100 \mathrm{C}$ is associated with bladder cancer progression and poor survival. Clin. Cancer Res. 11, 606-611 (2005).

19. Hori, O. et al. The receptor for advanced glycation end products (RAGE) is a cellular binding site for amphoterin. Mediation of neurite outgrowth and coexpression of rage and amphoterin in the developing nervous system. J. Biol. Chem. 270, 25752-25761 (1995).

20. Rauvala, H. \& Rouhiainen, A. RAGE as a receptor of HMGB1 (Amphoterin): roles in health and disease. Curr. Mol. Med. 7, 725-734 (2007).

21. Yan, S. D. et al. RAGE and amyloid-beta peptide neurotoxicity in Alzheimer's disease. Nature 382, 685-691 (1996).

22. Cecil, D. L. et al. Inflammation-induced chondrocyte hypertrophy is driven by receptor for advanced glycation end products. J. Immunol. 175, 8296-8302 (2005). 
23. van Zoelen, M. A. et al. Receptor for advanced glycation end products facilitates host defense during Escherichia coli-induced abdominal sepsis in mice J. Infect. Dis. 200, 765-773 (2009).

24. Algermissen, B., Sitzmann, J., LeMotte, P. \& Czarnetzki, B. Differential expression of CRABP II, psoriasin and cytokeratin 1 mRNA in human skin diseases. Arch. Dermatol. Res. 288, 426-430 (1996).

25. Hirono, K. et al. Expression of myeloid-related protein-8 and -14 in patients with acute Kawasaki disease. J. Am. Coll. Cardiol. 48, 1257-1264 (2006).

26. Hofmann, M. A. et al. RAGE mediates a novel proinflammatory axis: a central cell surface receptor for S100/calgranulin polypeptides. Cell 97, 889-901 (1999).

27. Sorg, C. The calcium binding proteins MRP8 and MRP14 in acute and chronic inflammation. Behring Inst. Mitt. 91, 126-137 (1992).

28. Pass, H. I. et al. Characteristics of nine newly derived mesothelioma cell lines. Ann. Thorac. Surg. 59, 835-844 (1995).

29. Phelps, R. M. et al. NCl-Navy Medical Oncology Branch cell line data base. J. Cell. Biochem. Suppl. 24, 32-91 (1996).
30. Suzawa, K. et al. Establishment and molecular characterization of cell lines from Japanese patients with malignant pleural mesothelioma. Oncol. Lett. 11, 705-712 (2016).

31. Shien, K. et al. Knockdown of the epidermal growth factor receptor gene to investigate its therapeutic potential for the treatment of non-small-cell lung cancers. Clin. Lung Cancer 13, 488-493 (2012).

32. Shien, K. et al. Prognostic impact of cancer stem cell-related markers in nonsmall cell lung cancer patients treated with induction chemoradiotherapy. Lung Cancer 77, 162-167 (2012).

33. Sato, H. et al. Targeting the miR-200C/LIN28B axis in acquired EGFR-TKI resistance non-small cell lung cancer cells harboring EMT features. Sci. Rep. 7, 40847 (2017).

34. Tanaka, N. et al. Frequent methylation and oncogenic role of microRNA-34b/c in small-cell lung cancer. Lung Cancer 76, 32-38 (2012). 